\title{
Mathematical models of dengue fever epidemiology: multi-strain dynamics, immunological aspects associated to disease severity and vaccines
}

\author{
Maíra Aguiar ${ }^{1}$ and Nico Stollenwerk ${ }^{2}$ \\ ${ }^{1}$ Centre for Mathematics and Applications, Faculty of Sciences and Technology, Nova University of Lisbon, 2829-516 \\ Caparica, Portugal \\ ${ }^{2}$ Centro de Matemática, Aplicações Fundamentais e Investigação Operacional, Faculdade de Ciências da Universidade \\ de Lisboa, Portugal. \\ ${ }^{1}$ Email: maira@ptmat.fc.ul.pt
}

\begin{abstract}
Epidemiological models formulated to describe the transmission of the disease and to predict future outbreaks, can become an interesting tool able to address specific public health questions, guiding public health authorities during implementation of disease control measures such as vector control and vaccination. In this paper, we survey a model framework for dengue fever epidemiology, the most important viral mosquitoborne disease in the world. Here, we discuss the role of number of subsequent infections versus detailed number of dengue serotypes included in the model framework and the human immunological aspects associated to disease severity, identifying the implications for model dynamics and its impact for vaccine implementation.
\end{abstract}

Keywords: Dengue fever, multi-strain models, vaccine, chaos, predictability.

\section{INTRODUCTION}

In recent years, mathematical modeling became an important tool for understanding infectious disease epidemiology and dynamics, leading to great advances for disease control, providing tools for assessing the potential impact of different public health intervention measures. Dengue fever epidemiological dynamics shows large fluctuations in disease incidence, and several mathematical models describing the transmission of dengue viruses have been proposed to explain the irregular behavior of dengue epidemics.

With about 400 million dengue infections occurring every year [1], the disease is now considered the most important viral mosquito-borne disease in the world. Dengue fever is caused by four antigenically related but distinct serotypes (DENV-1 to DENV-4). Infection by one serotype confers life-long immunity to that serotype and a period of temporary cross-immunity to other serotypes. Clinical response on exposure to a second serotype is complex and may depend on factors such as patient age, dengue serotype, sequence of infection and the interval between infection by one serotype and exposure to a second serotype. Sequential infection increases the risk of developing severe dengue, due to a process described as antibody-dependent enhancement $(\mathrm{ADE})$, where the pre-existing antibodies to previous dengue infection enhances the new infection [2], [3], [4].

Dengue fever epidemiological dynamics shows large fluctuations in disease incidence, and several mathematical models describing the transmission of dengue viruses have been proposed to explain the irregular behavior of dengue epidemics. Most of the models developed try to incorporate factors focusing on several different aspects of the disease combined with biological aspects of the vector and possible intervention measures, leading to a higher dimensional systems with hundreds of parameters that would need to be estimated from fewer available empirical data. The problem becomes mathematically difficult and to be predictive and not get lost in unnecessary components of a model, parsimony is often needed.

A careful review of deterministic dengue modeling was recently published [5], where two main approaches were considered, the vector-host and the (indirect) host-to-host transmission. In the first approach, the fluctuations in the mosquito dynamics and climate change are included and assumed to affect the disease transmission [6], [8], while in the second approach the effect of seasonality (mimicking the vectorial dynamics) appear to be essential to explain the intra-annual fluctuations in disease cases [9], [10]. Incidence maps have

Received August $29^{t h}, 2017$, Revised October $1^{\text {st }}, 2017$, Accepted for publication October $17^{\text {th }}, 2017$. Copyright $@ 2017$ Published by Bio Mathematical Society, e-ISSN: 2549-2896, DOI: 10.5614/cbms.2017.1.1.1 
also gained importance in the last years, giving visual insights on disease occurrence [1], [11] and vector abundance for example [12]; however, this surveillance tool is still difficult to be accurately used in the public health context of local disease intervention, given their static output (in contrast to the chaotic fluctuations in disease incidences), their complexity and the need of a constant good data input. The same difficulty is expected when dealing with more complex models including four different strains and vector dynamics individual based models and age-structure [13], [14].

In this survey we discuss some modeling framework recently used by the World Health Organization (WHO) to recommend the newly licensed dengue vaccine. We focus on the biological aspects of dengue fever epidemiology, identifying its implications for model dynamics and its impact for disease prevention and control.

\section{Multi-strain Dynamics, Antibody-Dependent Enhancement and Temporary Cross IMMUNITY}

Multi-strain dengue dynamics are generally modeled with extended SIR-type models and the combination of biological aspects such as temporary cross-immunity and the ADE effect have been studied by several authors. Modeling attention has first focused on higher viral load of hosts on secondary dengue infections due to ADE, but neglecting temporary cross-immunity, reporting deterministically chaotic attractors [6] and chaos desynchronization [15], [16] to explain the co-existence of the four dengue viral strains. Temporary cross-immunity was later incorporated in mathematical models as well, but again limiting the effect of ADE to increase the contribution of secondary cases to the force of infection, without further systematic investigation of the possible dynamical structures in these models [8], [13], [17], [18].

Aguiar et al. [19], [20] have investigated a two-infection dengue model, combining the effect of temporary cross-immunity and ADE, an extension of a model initially suggested and preliminarily analyzed in Ferguson et al. [6], where a rich dynamical behavior [7], from bifurcations up to deterministic chaos was found in wider and not previously described parameter regions, no longer needing to restrict the infectivity on secondary infection to be much higher than on primary infections (see Fig.1).

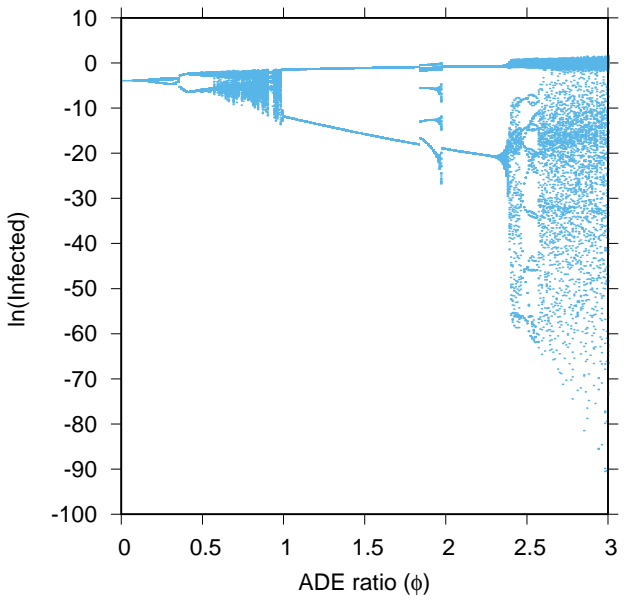

(a)

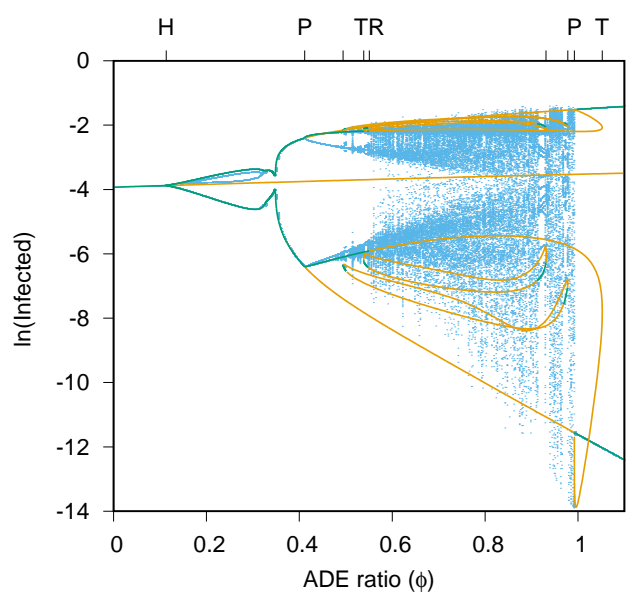

(b)

Fig. 1: Bifurcation diagrams of attractors changing with one model parameter, the ADE ratio $\phi$. Plotted are local maxima and minima of the attractors. a) For very small $\phi$ we observe fixed points, then periodic behaviour, followed by complex behaviour for phi smaller 1. Complex behaviour is found again, after periodic windows, for large ADE ratio phi around 3. b) Comparison of the numerical bifurcation diagram with an analysis of bifurcation software, AUTO, revealing the types of bifurcations, Hopf bifurcation $H$, pitchfork $P$ and torus bifurcation $T$ from limit cycles into tori from where more complex behaviour such deterministic chaos originates, until boundary crises again into periodic behaviour.

However, in order to be able to reproduce the yearly cycle in dengue incidence seasonal forcing and a low import of infected have to be included in the models [9]. The minimalistic model proposed in [9] has shown 
complex dynamics and qualitatively a good agreement between empirical data and model simulation. Note that for our deterministic system, Lyapunov exponents calculations were performed in order to quantify its prediction horizon, where beyond this prediction horizon we have unpredictability. For the chaotic region of $\phi=0.9$, the dominant Lyapunov exponent is 0.1118 giving $\approx 10$ years of prediction horizon in the monthly time series. In Fig. 2 we present two of the many different matching possibilities, able to describe a 5 year period of empirical data, from 1983 to 1994 and from 2002 to 2012, respectively, with a good qualitative (and even quantitative) agreement with our model simulation.

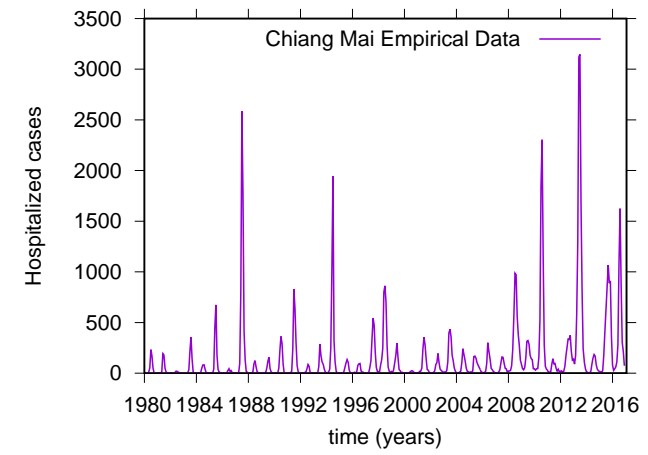

(a)

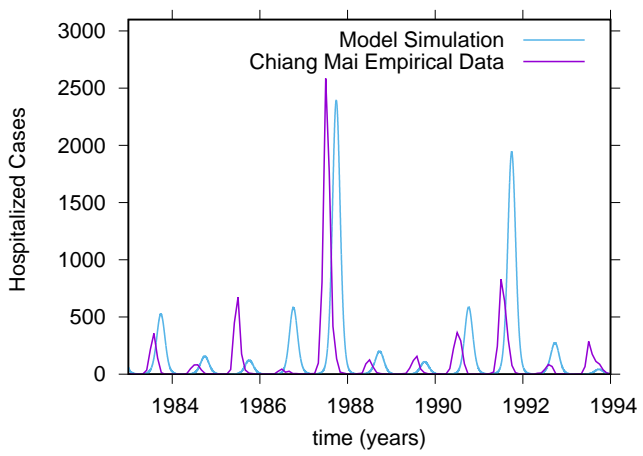

(c)

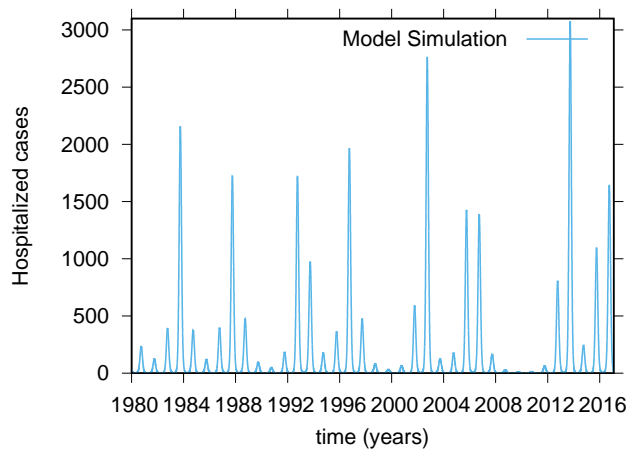

(b)

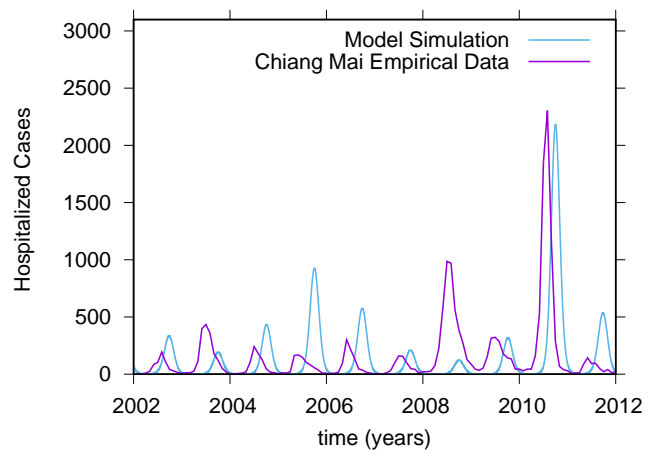

(d)

Fig. 2: Time series analysis. In a) the empirical data for hospital admission cases in Chiang Mai Province, North of Thailand. In $b$ ) the seasonal two-serotype model simulation, showing deterministic chaotic behavior confirmed by a positive Lyapunov exponent. The prediction horizon in the deterministic system is of approximately 10 years. The model shows similar pattern as the empirical data, with yearly irregular oscillations. The two-serotype model was validated and parametrized via data matching. As an example of the many different matching possibilities, we plot in c) and in d) 10 years of time series simulations with empirical data, from 1983 to 1994 and from 2002 to 2012 respectively.

Since then, the restrictive assumption of much higher contribution to the force of infection of secondary infectivity that was previously necessary to generate complex dynamics could be relaxed significantly when taking the temporary cross-immunity into account.

The findings of wide ranges of chaotic attractors have opened new ways to the analysis of existing data sets, indicating that deterministic chaos is much more important in multi-strain models than previously thought.

More than ever, temporary cross-immunity has turned out to be an important biological feature when modeling dengue fever epidemiology and has become an important ingredient for dengue modeling development, more often used in new modeling approaches [21]. 


\subsection{The two strain dengue model}

The minimalistic two-serotype model proposed by Aguiar et al. [9] is able to capture differences between primary and secondary dengue infection, including temporary cross-immunity and ADE. Only two possible infections are considered, primary and secondary, an assumption validated because of the low frequency of tertiary and quaternary infections among hospital cohorts [29]. The four-serotype model is represented in Fig. 3 using a state flow diagram.

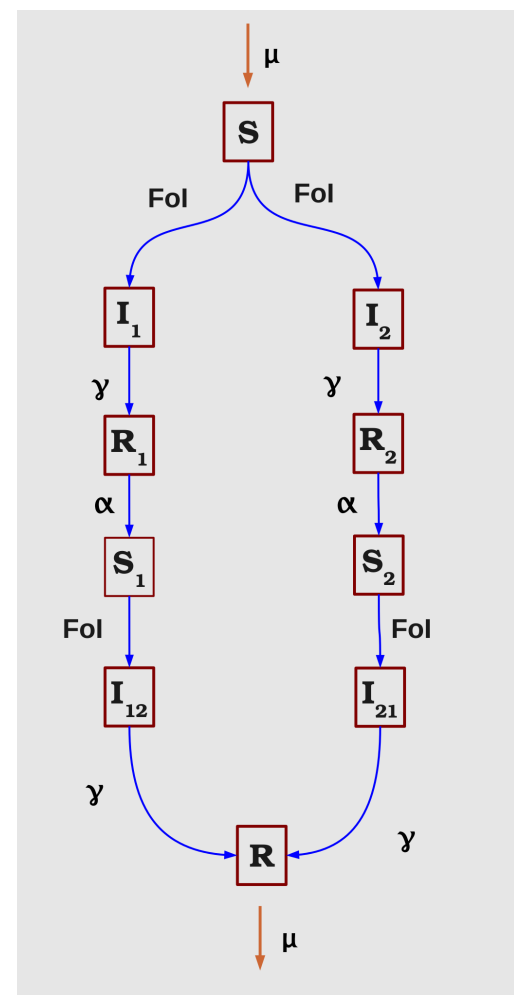

Fig. 3: State flow diagram for two-infection two-serotype dengue model. The Force of Infection (FoI) is explicitly given by $\beta\left(I_{1}+I_{2}+\rho N+\phi\left(I_{12}+I_{21}\right)\right.$, where $\beta$ takes the seasonal forcing into account as a cosine function $\beta(t)=$ $\beta_{0}(1+\eta \cdot \cos (\omega t))$, mimicking vector dynamics.

The complete system of ordinary differential equations for the seasonal multi-strain epidemiological model is shown in system (1).

The effects of the vector dynamics are only taken into account by the force of infection parameters in the SIR-type model, but not modeling this mechanisms explicitly [30]. The parameter $\beta$ takes the seasonal forcing into account as a cosine function.

The temporary cross-immunity period and the recovery rate are parametrized by $\alpha$ and $\gamma$ respectively. The population size $\mathrm{N}$ is constant and the demography (birth and death rates) is parametrized by $\mu$. Susceptible individuals can become infected also by meeting an infected individual from an external population contributing to the force of infection with an import parameter $\rho$. The parameter $\phi$ introduces the ADE ratio, i.e., secondary infection contribution to the force of infection. In this way, individuals with a primary infection and with a secondary infection transmit disease differently, with infection rate $\beta$ and $\phi \beta$ respectively.

The dynamics of the system is described as follows. For two different strains, 1 and 2 , we label the SIR classes for the hosts that have been infected naturally by individual strains. Individuals start as seronegative susceptibles $(S)$ and can acquire a natural first infection $\left(I_{1}\right)$ or $\left(I_{2}\right)$ with a specific dengue serotype. Individuals recover from a primary infection $\left(R_{1}\right)$ or $\left(R_{2}\right)$ and after a period of temporary cross-immunity, 
become seropositive (monotypic) susceptibles $\left(S_{1}\right)$ or $\left(S_{2}\right)$, now immune to one serotype and able to acquire a natural secondary dengue infection with a different serotype $\left(I_{12}\right)$ or $\left(I_{21}\right)$. Finally, individuals recover from a secondary infection $(R)$.

We assume that individuals experiencing a secondary infection are assumed to have higher risk (than primarily infected individuals) of developing clinically apparent and severe disease with hospitalization. For a more detailed description, see [9]. The parameter values are given in Table 1.

The minimalistic model successfully described large fluctuations observed in empirical outbreak data (see Fig. 2), estimating lower infection rate for secondary dengue infected individuals than for primary infected, anticipating results published recently in Duong et al. [31], where persons with unapparent dengue infections were more infectious to mosquitoes than clinically symptomatic patients.

$$
\begin{aligned}
& \dot{S}=-\frac{\beta(t)}{N} S\left(I_{1}+\rho \cdot N+\phi I_{21}\right)-\frac{\beta(t)}{N} S\left(I_{2}+\rho \cdot N+\phi I_{12}\right)+\mu(N-S) \\
& \dot{I_{1}}=\frac{\beta(t)}{N} S\left(I_{1}+\rho \cdot N+\phi I_{21}\right)-(\gamma+\mu) I_{1} \\
& \dot{I}_{2}=\frac{\beta(t)}{N} S\left(I_{2}+\rho \cdot N+\phi I_{12}\right)-(\gamma+\mu) I_{2} \\
& \dot{R_{1}}=\gamma I_{1}-(\alpha+\mu) R_{1} \\
& \dot{R_{2}}=\gamma I_{2}-(\alpha+\mu) R_{2} \\
& \dot{S}_{1}=-\frac{\beta(t)}{N} S_{1}\left(I_{2}+\rho \cdot N+\phi I_{12}\right)+\alpha R_{1}-\mu S_{1} \\
& \dot{S_{2}}=-\frac{\beta(t)}{N} S_{2}\left(I_{1}+\rho \cdot N+\phi I_{21}\right)+\alpha R_{2}-\mu S_{2} \\
& \dot{I_{12}}=\frac{\beta(t)}{N} S_{1}\left(I_{2}+\rho \cdot N+\phi I_{12}\right)-(\gamma+\mu) I_{12} \\
& \dot{I_{21}}=\frac{\beta(t)}{N} S_{2}\left(I_{1}+\rho \cdot N+\phi I_{21}\right)-(\gamma+\mu) I_{21} \\
& \dot{R}=\gamma\left(I_{12}+I_{21}\right)-\mu R
\end{aligned}
$$

TABLE I: Parameter set for the minimalistic two-strain dengue model.

\begin{tabular}{llll}
\hline Par. & Description & Values & Ref \\
\hline$N$ & population size & 1.6 million & {$[9]$} \\
$\mu$ & birth and death rate & $1 / 65 y$ & {$[9]$} \\
$\gamma$ & recovery rate & $52 y^{-1}$ & {$[9]$} \\
$\beta_{0}$ & infection rate & $2 \cdot \gamma$ & {$[9]$} \\
$\eta$ & degree of seasonality & 0.35 & {$[9]$} \\
$\rho$ & import parameter & 0 to $10^{-10}$ & {$[9]$} \\
$\alpha$ & temporary cross-immunity rate & $2 y^{-1}$ & {$[9]$} \\
$\phi$ & ratio of secondary infections & & \\
& contributing to force of infection & 0.9 & {$[9]$}
\end{tabular}




\subsection{The four strain dengue model}

Further extension of the previously studied model by Aguiar et al. [9] was performed, showing that the combination of temporary cross-immunity and ADE are the most important feature to drive the complex dynamics in the system, more than the detailed number of dengue serotypes to be added in the model [22]. For both models a qualitatively good result were obtained when comparing empirical data and model simulation, with similar patterns of the irregular behavior (see Fig. 5). The model is represented in Fig. 4 using a state flow diagram.

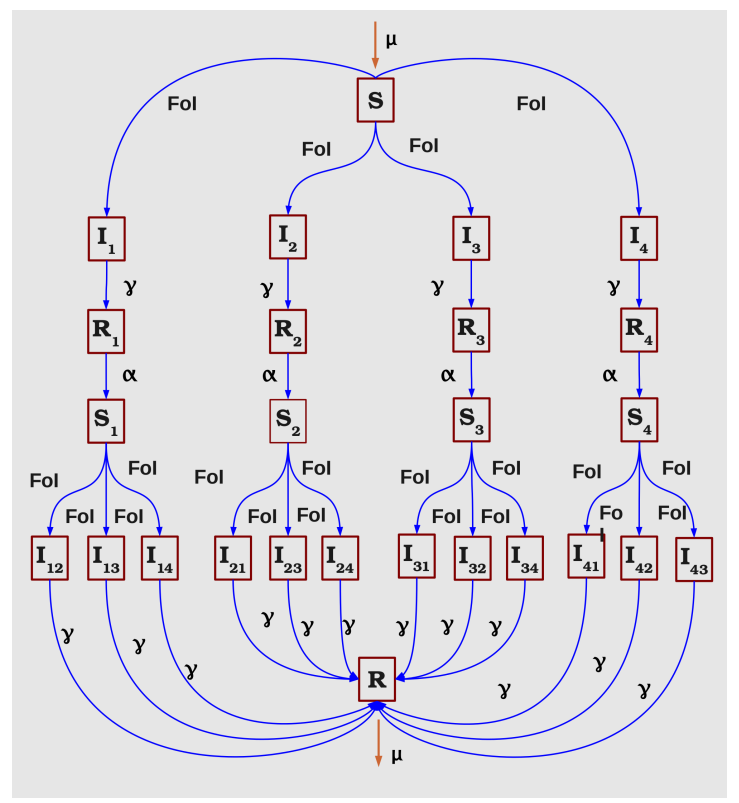

Fig. 4: State flow diagram for two-infection four-serotype dengue model. The Force of Infection (FoI) is explicitly given by $\beta\left(I_{1}+I_{2}+I_{3}+I_{4}+\rho N+\phi\left(I_{12}+I_{13}+I_{14}+I_{21}+I_{23}+I_{24}+I_{31}+I_{32}+I_{34}+I_{41}+I_{42}+I_{43}\right)\right.$, where $\beta$ takes the seasonal forcing into account as a cosine function $\beta(t)=\beta_{0}(1+\eta \cdot \cos (\omega t))$, mimicking vector dynamics.

The system dynamics are similar to the dynamics described for the two-serotype model. Again with only two possible infections but now with four strains, thus more infection possibilities. For a more detailed description of the four-serotype model, see [22].

The numerical bifurcation analysis has shown that chaotic dynamics appear to happen at the same parameter region of interest. For both models, a similar structure and the same order of magnitude of the dominant Lyapunov exponent were observed. This study has confirmed that the practical predictability of the system does not change significantly when considering two or four serotypes in the model assumptions, where the prediction horizon in a time series is in the same range for both models.

\section{THE IMPACT OF VACCINE IMPLEMENTATION}

Since 2016, a dengue vaccine, Dengvaxia, marketed by Sanofi Pasteur, is licensed for use in 19 endemic countries. Analysis of phase III trials' results suggested high rates of protection of vaccinated dengue seropositive individuals, but also high rates of hospitalizations during breakthrough dengue infections of persons who were vaccinated when seronegative [23]. The latter result indicates that Dengvaxia appears to induce dengue infection-enhancing antibodies (ADE) [24]. The availability of a vaccine for dengue with varying levels of efficacy against the different serotypes and potential counter-effects [25] makes extremely relevant the assessment of the potential effects of mass vaccination in endemic areas, as well as of different response policies in case of new vaccine licensure and implementation. 


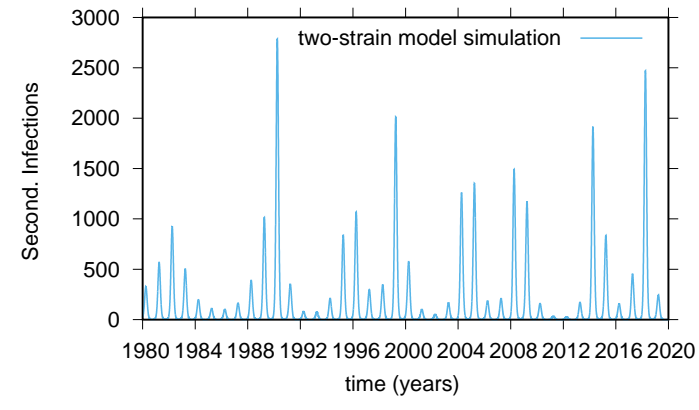

(a)

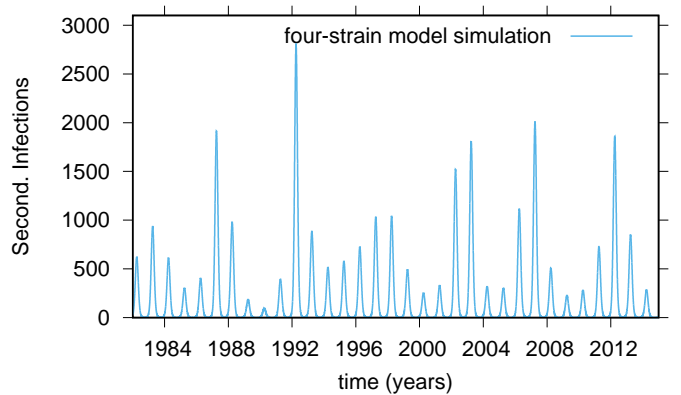

(b)

Fig. 5: Using the same parameter set, in a) the two-serotype model simulation and in b) the four-serotype model simulation. Parameter values are given in Table 1. For both models, similar patterns are observed with yearly irregular behavior and same prediction horizon estimated by the Lyapunov exponents' calculations. The effective dimension of the two serotype model is 9 while of the four serotype model 25. The law of parsimony that recommends selecting the hypothesis that makes the fewest assumptions, implies that the 9 dimensional two-serotype model would be the better candidate than the 25 dimensional four-serotype model to be analyzed, capturing the essential differences of primary versus secondary infection without needing to restrict the ADE effect to one or another region in parameter space.

Dengvaxia was recommended by the World Health Organization (WHO) Strategic Advisory Group of Experts (SAGE) on immunisation [23], based partially on modeling results [26], [27] estimating an overall reduction of $10-30 \%$ of dengue hospitalizations, over a period of 30 years, when this vaccine is yearly administrated in $80 \%$ of children (just) 9 years of age, living in high dengue endemic areas of more than $50 \%$ seroprevalence.

Opposing this prediction, Aguiar et al., have discussed the risks behind this vaccine recommendation [25], [32], after analyzing an age structured model [28]. Using the public available vaccine trial data, vaccine efficacy was estimated via the Bayesian approach [33], predicting a significant reduction of hospitalizations only when the vaccine is given to seropositive individuals [28].

\subsection{Vaccine efficacy estimation via the Bayesian approach}

Using the data for the newly licensed dengue vaccine trial in the Asian-Pacific region (CYD14), with participants age between 2-14, as reported in [34], and for the Latin America (CYD15), which has enrolled individuals from 9-14 years of age, as reported in [35], we have estimated the vaccine efficacy (VE) via the Bayesian approach [36], [37], to obtain explicitly a probability $p\left(k \mid I_{v}, I_{c}\right)$ for the vaccine efficacy $k$ with infected individuals (with $I_{v}$ in the vaccine group and $I_{c}$ in the placebo group). We obtained a statistical description of the vaccine trial data that were in very good agreement with the published results for the vaccine efficacy for confirmed dengue cases during the year 1 and 2 of the trials. The Bayesian estimates of the vaccine efficacy for confirmed dengue cases during the years 1-2 in the CYD14 and CYD15 trials is shown in Table 2. Note that the uninformed Bayesian prior might have a slight influence mainly in the confidence interval values.

TABLE II: Bayesian estimates of the VE for confirmed dengue cases during the years 1-2 in the CYD14 and CYD15 trials

\begin{tabular}{llll}
\hline & Published Estimates & Our Estimates & Ref \\
\hline Latin America Region (CYD15) & $56.5 \%$ 95\%-CI (43.8;66.4) & $56.56 \% 95 \%$-CI $(44.4 ; 66.2)$ & {$[28],[34]$} \\
Asian-Pacific Region (CYD14) & $60.8 \% 95 \%$-CI (52.0;68) & $60.76 \% 95 \%$-CI $(52.2 ; 70.5)$ & {$[28],[35]$} \\
Overall VE (CYD14+CYD15) & - & $59.2 \% 95 \%$-CI $(52.4 ; 66)$ & {$[28],[33]$} \\
& & & \\
\hline
\end{tabular}

Using the combined data from Sanofi-Pasteurs dengue vaccine trials (CDY14 and CYD15) we estimated the overall vaccine efficacy, via the Bayesian approach, to obtain a probability for the combined vaccine efficacy 
based on the empirical data from Asian-Pacific and the Latin American trials [34], [35]. The Bayesian estimate of the combined vaccine efficacy trials is $\mathrm{k}=59.2 \%$ with a $95 \%$-CI of $(52.4 ; 65.0)$ as shown in Table 2.

Using the same methodology, the specific vaccine efficacy against each dengue serotypes was analyzed, revealing a scenario where any possible common efficacy for all serotypes is statistical excluded.

While results from years 12 have demonstrated an intermediate efficacy for confirmed dengue cases, in the year 3, vaccine efficacy for hospitalized dengue cases was found to be considerably smaller for seronegative individuals at baseline (individuals that have never been infected by a dengue virus prior to the vaccine trial) than for individuals who were seropositive at baseline (individuals that have been infected by a dengue virus prior to the vaccine trial) [23], [38]. Moreover, a negative vaccine efficacy was estimated, for the years 3 and 4 of the CYD14 trial, with vaccine disease-enhancement in younger children, which were in its majority seronegatives [28], [32]. For a more detailed calculation, see [28], [37].

Note that the CYD14 is of interest given the possibility to evaluate both age groups, individuals under and older 9 years of age. The Bayesian estimates of the vaccine efficacy for hospitalized dengue cases during the years 3-4 in the CYD14 trial is shown in Table 3.

TABLE III: Bayesian estimates of the VE for hospitalized dengue cases during the years 3-4 in the CYD14 trial

\begin{tabular}{|c|c|c|c|}
\hline CYD14 trial (age groups) & Year & VE estimation & Ref \\
\hline $2-5$ years & 3 & $-520 \% 95 \%-$ CI $(-5089 ;-14.1)$ & [23], [28], [32], [38] \\
\hline $2-5$ years & 4 & $-40.8 \% 95 \%$-CI $(-253.5 ; 36.8)$ & {$[23],[28],[32],[38]$} \\
\hline $6-8$ years & 3 & $60 \%$ 95\%-CI $(-86 ; 92.0)$ & [23], [28], [32], [38] \\
\hline $6-8$ years & 4 & $0 \%$ 95\%-CI $(-153 ; 57)$ & [23], [28], [32], [38] \\
\hline $2-8$ years & 3 & $-55.4 \% 95 \%$-CI $(-318.1 ; 33.3)$ & [23], [28], [32], [38] \\
\hline $2-8$ years & 4 & $-18.1 \% 95 \%$-CI $(-117,1 ; 32,3)$ & {$[23],[28],[32],[38]$} \\
\hline $9-11$ & 3 & $2.5 \% 95 \%$-CI $(-330.2 ; 73.2)$ & {$[23],[28],[32],[38]$} \\
\hline $9-11$ & 4 & $-92.4 \% 95 \%$-CI $(-160.3 ; 36.8)$ & [23], [28], [32], [38] \\
\hline $11-14$ & 3 & $75 \% 95 \%-C I(-74 ; 98)$ & {$[23],[28],[32],[38]$} \\
\hline $11-14$ & 4 & $65 \% 95 \%$-CI $(0 ; 89)$ & [23], [28], [32], [38] \\
\hline $9-14$ & 3 & $42.9 \% 95 \%$-CI $(-59.5 ; 79.2)$ & {$[23],[28],[32],[38]$} \\
\hline $9-14$ & 4 & $27.2 \% 95 \%$-CI $(-49.9 ; 63.5)$ & [23], [28], [32], [38] \\
\hline
\end{tabular}

\section{MODELING VACCINE IMPLEMENTATION}

To model the future vaccine impact, the minimalistic two-strain dengue model [9] was extended. An age structured model was developed based on the WHO recommendation to vaccinate persons age 9-45 years in dengue endemic countries. The model is represented in Fig 4 using a state flow diagram, where the population consists of a mixture of seronegative and seropositive individuals and only a percentage of individuals 9-45 years of age are vaccinated.

Similarly to the assumptions used in the WHO-SAGE modeling exercise, the model is constructed such the vaccine in seronegative individuals acts like a asymptomatic natural (first) infection providing a short-term protection against the four dengue serotypes. As the immunity wains, those individuals have high probability of symptomatic and severe disease when acquiring a natural primary infection. Vaccine efficacy for seropositive individuals was assumed to be close to $100 \%$, preventing dengue infections and hospitalization of monotypic dengue immune individuals. Although more data in respect to individual sero-status prior to vaccination are still needed, for this analysis we have assumed that individuals of any age who have experienced at least one dengue virus infection will benefit from vaccination but when seronegative, an increased risk of vaccine disease enhancement exists.

Insights from our mathematical modeling have shown that high rates of reduction of hospitalizations can be achieved when vaccinating only seropositive individuals. While dengue infections of seropositive individuals will be prevented, vaccination of seronegative persons increases hospitalization (see Fig. 5). The detailed modeling analysis can be found in [28]. The model parameters are shown in Table 4.

\section{Discussion}

In this survey we analyzed a modeling framework and assumptions recently used to asses the impact of the newly licensed dengue vaccine. 


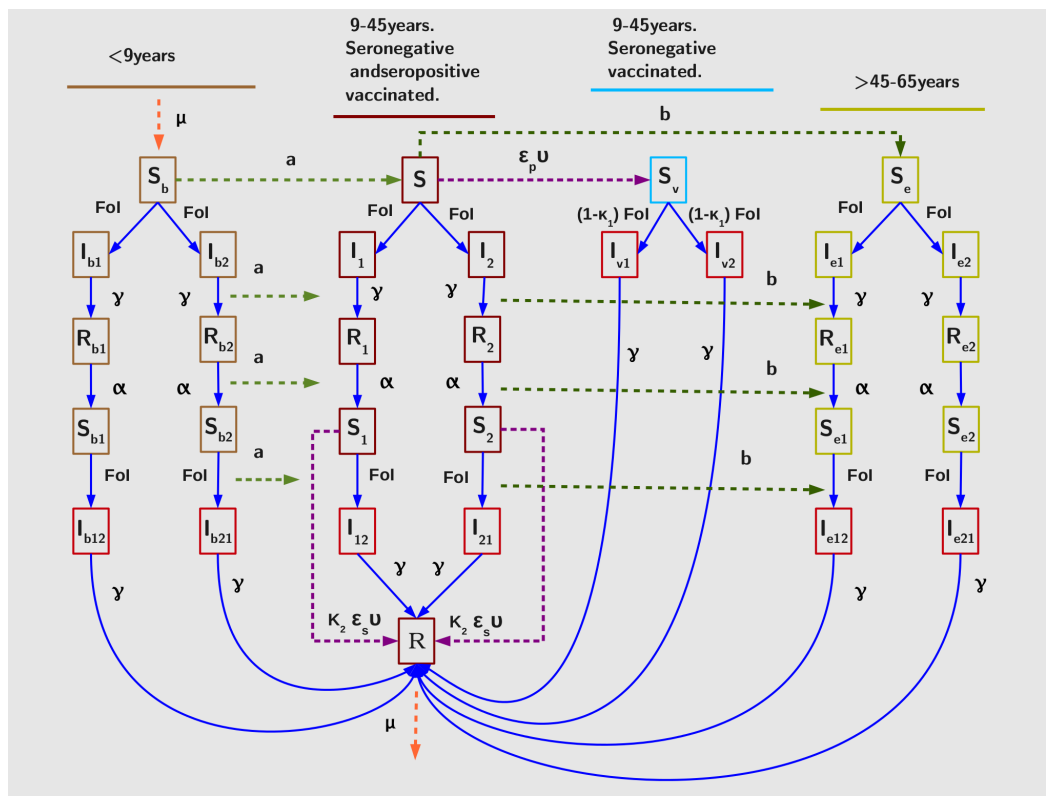

Fig. 6: Flow diagram for age-structured two-infection two-serotype dengue model with vaccination. Hospitalizations are only possible in the classes marked in red. The Force of Infection (FoI) is explicitly given by $\beta\left(I_{1}+I_{2}+I_{b 1}+I_{b} 2+\right.$ $\left.I_{e} 1+I_{e} 2+\rho N+\phi\left(I_{12}+I_{21}+I_{b 12}+I_{b 21}+I_{e 12}+I_{e 21}\right)+\phi_{v}\left(I_{v 1}+I_{v 2}.\right)\right)$.

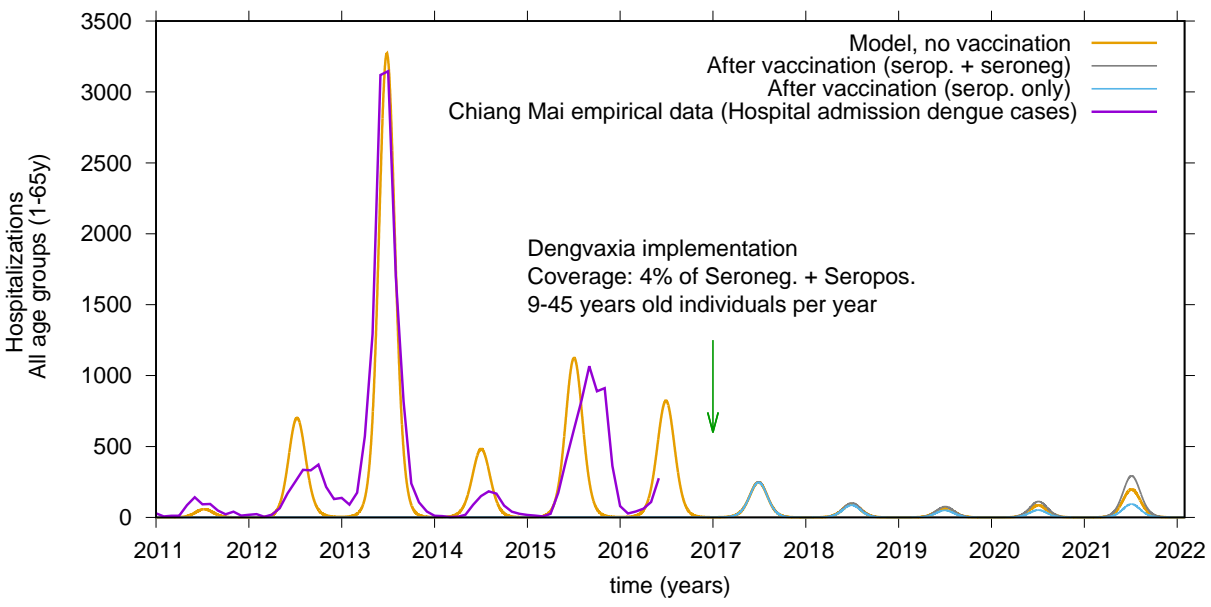

Fig. 7: Model simulation of hospitalizations in a population between 165 year-old. The last 5 years of available empirical data (in purple) were matched with the model simulation prior to vaccination (in orange) and prediction was made for the following 5 years, after vaccine implementation covering yearly $4 \%$ of the target group between 9-45 years of age, from 2016 to 2021. Hospitalizations occurring after yearly vaccination of both seropositives and seronegatives, ages 9-45 years, are shown as a gray line. However, when restricting this vaccine to seropositive only, 9-45 years of age, high rates of reduction of hospitalizations can be achieved (in blue). For this simulation, no extra risk of vaccine disease enhancement, compared with hospitalizations of individuals hospitalized when acquiring a natural secondary infection, was considered. However, if that would be the case, the number of hospitalization without population screening would be much larger (see [28]). 
TABLE IV: Estimated parameter set via data matching

\begin{tabular}{|c|c|c|c|}
\hline Par. & Description & Values & Ref \\
\hline$N$ & population size & 1.65 million inhabitants & [9], [28] \\
\hline$\mu$ & birth and death rate & $1 / 65 y$ & [9], [28] \\
\hline$\gamma$ & recovery rate & $52 y^{-1}$ & [9], [28] \\
\hline$\beta$ & effective infection rate & $2 \gamma$ & [9], [28] \\
\hline$\eta$ & degree of seasonality & 0.35 & [9], [28] \\
\hline$\varphi$ & phase of seasonality & $3 / 12 y$ & [9], [28] \\
\hline$\rho$ & import parameter & $10^{-8}$ & [9], [28] \\
\hline $\begin{array}{l}\alpha \\
\phi=\phi_{v}\end{array}$ & $\begin{array}{l}\text { temporary cross-immunity rate } \\
\text { ratio of secondary infections }\end{array}$ & $2 y^{-1}$ & [9], [28] \\
\hline & contributing to force of infection & 0.9 & {$[2],[3],[4],[9],[28],[31]$} \\
\hline & vaccination rate & $1 / 1 y$ & {$[28],[34],[35],[38]$} \\
\hline $\begin{array}{l}\epsilon_{p}=\epsilon_{s} \\
k_{1}\end{array}$ & $\begin{array}{l}\text { proportion of vaccinated population } \\
\text { estimated vaccine efficacy }\end{array}$ & $4 \%$ per year & [28] \\
\hline \multirow{3}{*}{$k_{2}$} & for hospitalization under $9 y$ & -0.55 & [28], [37], [38] \\
\hline & estimated vaccine efficacy & & \\
\hline & for hospitalization older $9 \mathrm{y}$ & 0.9 & {$[28],[38]$} \\
\hline
\end{tabular}

Starting from a basic two-serotype SIR-type model for the host population, model extensions were investigated and the role of the detailed number of dengue serotypes to be included into the framework was discussed. The minimalistic model proposed by Aguiar et al. [9] in its simplicity as a good model to be analyzed, giving the expected complex behavior to explain the fluctuations observed in empirical data. It can capture the essential differences of primary versus secondary infection without needing to restrict the ADE effect to one or another region in parameter space. Moreover, the model has shown a qualitatively good result when comparing empirical incidence data and model simulations, anticipating results published recently in Duong et al. [31], where persons with unapparent dengue infections were more infectious to mosquitoes than clinically symptomatic patients.

Focusing on the biological aspects of dengue fever epidemiology and its implications for model dynamics, the analysis of the four-serotype dengue model [22] have shown that the combination of temporary crossimmunity and ADE are the most important feature to drive the complex dynamics in the system, with chaotic behavior appearing at the same parameter region of interest. By using the same parameter set, prediction horizon was observed to be similar for both models, the two and four-serotypes models.

To be predictive and not get lost in unnecessary components of a model, parsimony is often needed. For vaccine implementation, the two-serotype model with two possible infections was selected and extended. Using similar assumptions as some models in the WHO-SAGE modeling consortia, our model [28] was constructed assuming that individuals of any age who have experienced at least one dengue virus infection would benefit from vaccination but when seronegative, an increased risk of vaccine disease enhancement would be experienced.

Vaccine efficacy in preventing hospitalized dengue cases was estimated using the data available in [23], [38], via the Bayesian approach [36], [37]. Chaotic behavior exists in many natural systems and in the case of our deterministic system, whose behavior can in principle be predicted for approximately 10 years, model was parameterized by matching the last 5 years of available empirical hospitalized dengue data for Chiang Mai with model simulations and predictions for disease outcome after vaccine implementation were evaluated for a period of 5 years. This time-frame seams to be acceptable, since neither seroprevalence nor vaccine efficacy are static quantities, and therefore, a prediction of 30 years raises questions of the validity of the recommendation by the WHO-SAGE.

Our results suggested that reserving vaccine for seropositive individuals should provide a high level of protection whereas vaccinating indiscriminately could increase the number of hospitalizations also on the population level.

We are concerned about seronegatives at any age receiving this vaccine and a question that needs to be addressed at all prospective vaccination sites is which screening test to choose to identify seropositives, since ELISA IgG tests vary in specificity [25], [32]. 


\section{ACKNOWLEDGEMENTS}

This work was supported by Strategic Project UID/MAT/00297/2013 (Centro de Matemática e Aplicaçẽs, Universidade Nova de Lisboa)

\section{REFERENCES}

[1] Bhatt, S., Gething, P.W., Brady, O.J., Messina, J.P., Farlow, A.W., Moyes, C.L., Drake, J.M., Brownstein, J.S., Hoen, A.G., Sankoh, O., Myers, M.F., George, D.B., Jaenisch, T., Wint, G.R.W., Simmons, C.P., Scott, T.W., Farrar, J.J., and Hay, S.I. (2013). The global distribution and burden of dengue. Nature; 496 504-507.

[2] Halstead, S.B. (2003). Neutralization and antibody-dependent enhancement of dengue viruses. Advances in Virus Research; 60:421-467.

[3] Guzman, M.G., Halstead, S.B., Artsob, H., Buchy, P., Farrar, J., Gubler, D.J., Hunsperger, E., Kroeger, A., Margolis, H.S., Martnez, E., Nathan, M.B., Pelegrino, J.L., Simmons, C., Yoksan, S. and Peeling, R.W. (2010). Dengue: a continuing global threat. Nature Reviews Microbiology, 8, S7-S16.

[4] Dejnirattisai, W., Jumnainsong, A., Onsirisakul, N., Fitton, P., Vasanawathana, S., Limpitikul, W., Puttikhunt, C., Edwards, C., Duangchinda, T., Supasa, S., Chawansuntati, K., Malasit, P., Mongkolsapaya, J. and Screaton, G. (2010). Cross-Reacting Antibodies Enhance Dengue Virus Infection in Humans. Science, 328, 745-748.

[5] Andraud, M., Hens, N., Marais, C. and Beutels, P. (2012). Dynamic Epidemiological Models for Dengue Transmission: A Systematic Review of Structural Approaches. PLoS ONE 7(11): e49085.

[6] Ferguson, N., Anderson, R. and Gupta, S. (1999). The effect of antibody-dependent enhancement on the transmission dynamics and persistence of multiple-strain pathogens. Proc. Natl. Acad. Sci. USA; 96:790-94.

[7] Aguiar, M., Stollenwerk, N., and Kooi, B. (2009). Torus bifurcations, isolas and chaotic attractors in a simple dengue fever model with ADE and temporary cross immunity. Int. J. Comput. Math.; 86:1867-77.

[8] Wearing, H.J. and Rohani, P. (2006). Ecological and immunological determinants of dengue epidemics. Proc. Natl. Acad. Sci. USA; 103:11802-11807.

[9] Aguiar, M., Ballesteros, S., Kooi, B.W. and Stollenwerk, N. (2011). The role of seasonality and import in a minimalistic multistrain dengue model capturing differences between primary and secondary infections: complex dynamics and its implications for data analysis. J. Theor. Biol.; 289:181-196.

[10] Aguiar, M., Stollenwerk, N. and Kooi, W. B. (2012). Scaling of stochasticity in dengue hemorrhagic fever epidemics. Math. Model. Nat. Phenom., 7, 1-11.

[11] Messina, J.P., Brady, O.J., Pigott, D.M., Golding, N., Kraemer, M.U.G., Scott, T.W., Wint, G.R.W., Smith, D.L. and Hay, S.I. (2015). The many projected futures of dengue. Nature Reviews Microbiology ; 13: 230239.

[12] Kraemer, M.U.G., Sinka, M.E., Duda, K.A., Mylne, A.Q.N., Shearer, F.M., Barker, C.M., Moore, C.G., Carvalho, R.G., Coelho, G.E., Van Bortel, W., Hendrickx, G., Schaffner, F., Elyazar, I.R., Teng, H.-., Brady, O.J., Messina, J.P., Pigott, D.M., Scott, T.W., Smith, D.L., William Wint, G.R., Golding, N. and Hay, S.I. (2015). The global distribution of the arbovirus vectors Aedes aegypti and Ae. Albopictus. ELife; 4:e08347.

[13] Recker, M., Blyuss, K.B., Simmons, C.P., Hien, T.T., Wills, B., Farrar, J. and Gupta, S. (2009). Immunological serotype interactions and their effect on the epidemiological pattern of dengue. Proc. R. Soc. B 276, 25412548.

[14] Coudeville, L., Garnett, G.P.. (2012). Transmission Dynamics of the Four Dengue Serotypes in Southern Vietnam and the Potential Impact of Vaccination. PLoS ONE 7(12): e51244.

[15] Schwartz, I.B., Shaw, L.B., Cummings, D.A.T., Billings, L., McCrary, M., Burke, D.S., (2005). Chaotic desynchronization of multi-strain diseases. Phys. Rev. E 72, 066201066206.

[16] Billings, L., Schwartz, I.B., Shaw, L.B., McCrary, M., Burke, D.S. and Cummings, D.A.T. (2007). Instabilities in multiserotype disease models with antibody-dependent enhancement. J. Theor. Biol. 246, 1827.

[17] Nagao, Y., Koelle, K., (2008). Decreases in dengue transmission may act to increase the incidence of dengue hemorrhagic fever. Proc. Natl. Acad. Sci. USA 105, 22382243.

[18] Wikramaratna, P.S., Simmons, C.P., Gupta, S. and Recker, M. (2010). The effects of tertiary and quaternary infections on the epidemiology of dengue. PLoS ONE 5, e12347.

[19] Aguiar, M., Stollenwerk, N., (2007). A new chaotic attractor in a basic multi-strain epidemiological model with temporary crossimmunity. arXiv:0704.3174v1 [nlin.CD].

[20] Aguiar, M., Kooi, B.W., Stollenwerk, N., (2008). Epidemiology of dengue fever: a model with temporary cross-immunity and possible secondary infection shows bifurcations and chaotic behaviour in wide parameter regions. Math. Model. Nat. Phenom. 4, 4870.

[21] Reich, N.G., Shrestha, S., King, A.A., Rohani, P., Lessler, J., Kalayanarooj, S., Yoon, I.-., Gibbons, R.V., Burke, D.S. and Cummings, D.A.T. (2013). Interactions between serotypes of dengue highlight epidemiological impact of cross-immunity. J R Soc Interface 10: 20130414.

[22] Aguiar, M., Kooi, B.W., Rocha, F., Ghaffari, P. and Stollenwerk, N. (2013). How much complexity is needed to describe the fluctuations observed in dengue hemorrhagic fever incidence data? Ecol. Complex.; 16:31-40. 
[23] World Health Organization Strategic Advisory Group of Experts (SAGE) on Immunization. Background paper on Dengue Vaccines prepared by the SAGE working group on dengue vaccines and the WHO secretariat. (2016). Retrieved from http : //www.who.int/immunization/sage/meetings/2016/april/presentations background docs/en/

[24] Scott B. Halstead. (2016). Protective and Immunological Behavior of Yellow Fever Dengue Chimeric Vaccine. Vaccine, 34(14), 1643-647.

[25] Aguiar M, Stollenwerk N, Halstead SB. (2016). The risks behind Dengvaxia recommendation. Lancet Infect Dis. $2016 ; 16: 882$.

[26] Ferguson N, Rodrguez-Barraquer I, Dorigatti I, et al. (2016). Benefits and risks of the Sanofi-Pasteur dengue vaccine: modeling optimal deployment. Science. 2016;353:10331036.

[27] Flasche, S., Jit, M., Rodrguez-Barraquer, I., Coudeville, L., Recker, M., Koelle, K., Milne, G., Hladish, T.J., Perkins, T.A., Cummings, D.A.T., Dorigatti, I., Laydon, D.J., Espaa, G., Kelso, J., Longini, I., Lourenco, J., Pearson, C.A.B., Reiner, R.C., Miery-Tern-Romero, L., Vannice, K. and Ferguson, N. (2016) The Long-Term Safety, Public Health Impact, and Cost-Effectiveness of Routine Vaccination with a Recombinant, Live-Attenuated Dengue Vaccine (Dengvaxia): A Model Comparison Study. PLoS Med 13(11): e1002181.

[28] Aguiar M, Stollenwerk N, Halstead SB. (2016). The impact of the newly licensed dengue vaccine in endemic countries. PLoS Negl Trop Dis. 2016;10(12):e0005179. doi:10.1371/journal.pntd.0005179

[29] Gibbons, R.V., Kalanarooj, S., Jarman, R.G., Nisalak, A., Vaughn, D.W., Endy, T.P., Mammen Jr., M.P. and Srikiatkhachorn, A (2007). Analysis of repeat hospital admissions for dengue to estimate the fre- quency of third or fourth dengue infections resulting in admissions and dengue hemorrhagic fever, and serotype sequences. Am. J. Trop. Med. Hyg., 77(5), 910913.

[30] Rocha F., Aguiar M.,Souza M., and Stollenwerk N.. (2013). Time-scale separation and center manifold analysis describing vectorborne disease dynamics, Int. J. Comput. Math., 90(10), 21052125.

[31] Duong, V., Lambrechts, L., Paul, R.E., Ly, S., Lay, R.S., Long, K.C., Huy, R., Tarantola, A., Scott, T.W., Sakuntabhai, A. and Buchy, P. (2015). Asymptomatic humans transmit dengue virus to mosquitoes. Proc. Natl. Acad. Sci. USA, 112, 14688-93.

[32] Maíra Aguiar, Scott B. Halstead and Nico Stollenwerk. (2017). Consider stopping dengvaxia administration without immunological screening. Expert Review of Vaccines, 16 (4), 301-302.

[33] Maíra Aguiar, Luis Mateus and Nico Stollenwerk. (2016). The currently best estimate for worldwide dengue vaccine efficacy. AIP Conference Proceedings, 1738, 390014.

[34] Capeding, M.R., Tran, N.H., Hadinegoro, S.R.S., Ismail, H.I.H.M., Chotpitayasunondh, T., Chua, M.N., Luong, C.Q., Rusmil, K., Wirawan, D.N., Nallusamy, R., Pitisuttithum, P., Thisyakorn, U., Yoon, I.-., Van Der Vliet, D., Langevin, E., Laot, T., Hutagalung, Y., Frago, C., Boaz, M., Wartel, T.A., Tornieporth, N.G., Saville, M. and Bouckenooghe, A. (2014). Clinical efficacy and safety of a novel tetravalent dengue vaccine in healthy children in Asia:a phase 3, randomised, observer-masked, placebo-controlled trial. Lancet; 384:1358-65.

[35] Villar, L., Dayan, G.H., Arredondo-Garca, J.L., Rivera, D.M., Cunha, R., Deseda, C., Reynales, H., Costa, M.S., Morales-Ramrez, J.O., Carrasquilla, G., Rey, L.C., Dietze, R., Luz, K., Rivas, E., Montoya, M.C.M., Supelano, M.C., Zambrano, B., Langevin, E., Boaz, M., Tornieporth, N., Saville, M. and Noriega, F. (2015). Efficacy of a tetravalent dengue vaccine in children in Latin America. New Engl. J. Med. ; 372:113-123.

[36] Stollenwerk, N., Aguiar, M., Ballesteros, S., Boto, J., Kooi, W. B., and Mateus, L. (2012). Dynamic noise, chaos and parameter estimation in population biology, J. Royal Soc. Interface Focus 2, 156169.

[37] Mateus, L., Stollenwerk, N., and Zambrini, J.C. (2013) Stochastic Models in Population Biology: From Dynamic Noise to Bayesian Description and Model Comparison for Given Data Sets, Int. Journal. Computer Math. 90, 21612173.

[38] Hadinegoro S.R., Arredondo-Garcia J.L., Capeding M.R., Deseda C., Chotpitayasunondh T., Dietze R., et al. (2015). Efficacy and Long-Term Safety of a Dengue Vaccine in Regions of Endemic Disease. N. Engl. J. Med., 373, 1195206. 